\title{
Vieles wird anders!?
}

\author{
Jochen Schiewe ${ }^{1}$
}

Accepted: 21 May 2021 / Published online: 2 June 2021

(c) The Author(s) 2021

Wenn wir der Bundeskanzlerin Glauben schenken möchten, werden alle erwachsenen Bürgerinnen und Bürger bis zu den Bundestagswahlen im September 2021 (nebenbei: damit auch bis zum Deutschen Kartographie Kongress) geimpft sein können. Es ist also an der Zeit, sich Gedanken über die „Zeit nach Corona “ zu machen. „Vieles wird anders! “, behaupten und hoffen manche, während andere damit rechnen, dass wir schnell wieder in alte Gewohnheiten verfallen. Wahrscheinlich wird sich die Realität irgendwo dazwischen einpendeln.

Damit stellt sich auch die Frage, wo sich die DGfK mit ihren Aktivitäten und Themen in der Zeit nach Corona einpendeln wird. Ein zentraler Punkt hierbei wird die Gestaltung unserer Veranstaltungsformate sein. Ich denke, dass es gegenüber der Vor-Corona-Zeit ein deutlich breiteres Angebot geben wird, aus dem sich jedes Mitglied eine passende Mischung heraussuchen kann:

- Präsenzangebote: Die Corona-Zeit hat auch (hoffentlich...) zu einem stärkeren Klima-Bewusstsein geführt. Außerdem haben viele Arbeitgeber gemerkt, dass Zoom-Lizenzen günstiger als Reisebudgets sind. Beide Aspekte führen dazu, dass es weniger Dienstreisen — und damit auch eine geringere Nachfrage nach Präsenzveranstaltungen geben wird. Damit diese überhaupt eine Daseinsberechtigung haben werden, müssen sie einen Mehrwert bieten - für jede Einzelperson, aber auch für Firmen und Behörden, die das Budget zur Verfügung stellen. Für die Einzelperson besteht der Mehrwert gegenüber den digitalen Treffen sicherlich in der Möglichkeit des persönlichen Austausches. Folglich müssen wir bei Tagungen wie dem Deutschen Kartographie Kongress (DKK) mehr Raum und Zeit für das "Socializing“" vorsehen. Gleichzeitig sind mehr Themenschwerpunkte notwendig, z. B. in Form von Workshops,

Jochen Schiewe

jochen.schiewe@hcu-hamburg.de

1 Hamburg, Germany die eine umfassendere und gezieltere Weiterbildung ermöglichen als nur durch ein oder zwei passende Präsentationen aus einem klassischen Vortragsprogramm. Einen besonderen Fall stellen noch die Präsenz-Veranstaltungen der Sektionen dar-diese können und sollten m. E. in Zukunft aufgrund der kurzen Anreisen und kompakter Programme wieder mehr Bedeutung erlangen.

- Digitale Angebote: Die Vorteile digitaler Veranstaltungsformate-wie der monatlichen CartoCafés oder CartoHacks der DGfK-liegen auf der Hand: Sie sind günstig, räumlich flexibel und zeitlich effizient. Andererseits fehlt es aber noch an Kommunikations-Möglichkeiten bzw. deren konsequenter Anwendung und Gewöhnung. Im CartoCafé testen wir gerade eine einfache Form, bei der man sich auch nach dem Café in Kleingruppen treffen und unterhalten kann. Es ist absehbar, dass noch ansprechendere und trotzdem einfache technische Lösungen entwickelt werden. Und auch wenn der reale persönliche Kontakt weiter fehlen wird: Wir werden $\mathrm{CartoCa}$ fés und CartoHacks auch nach Corona sicherlich weiter anbieten!

- Hybride Angebote: Die Durchführung von Veranstaltungen als gleichzeitige Vor-Ort- und digitale Variante ist derzeit ein häufig genannter Trend. Allerdings ist die technische und organisatorische Umsetzung aufwändig und teuer- und bedarf sicherlich noch weiterer Entwicklungen und Erfahrungen. Letztere werden wir voraussichtlich schon beim DKK $2021 \mathrm{im}$ September sammeln können, der im Rahmen der-hybridenINTERGEO stattfinden wird.

Es ist leicht vorhersehbar, dass uns - auch nach Coronadie kartographischen Themen nicht ausgehen werden. Uns ist einmal mehr bewusst gemacht worden, dass wir in einer globalisierten Gesellschaft leben, in der Gesundheit, Klima und viele andere Bereiche grenzenlos betrachtet werden müssen. Das hat u. a. zur Folge, dass der Trend zu „Big Data " voranschreiten wird. Nicht nur die künstliche Intelligenz wird dadurch noch bedeutender werden-auch die Notwendigkeit von mehr und besseren Visualisierungen. 
Dass Kartographinnen und Kartographen als DIE Expertinnen und Experten für die Nutzbarmachung von solchen großen, heterogenen und unsicheren Datenbeständen gefragt sind, steht außer Frage-ebenso, dass es noch genügend Entwicklungspotenzial in diesem Kontext gibt, z. B. bei zeitabhängigen, kollaborativen oder individualisierten Darstellungen. Damit wird auch die Notwendigkeit zum fachlichen Austausch weiter ansteigen-im Rahmen von Veranstaltungen, aber natürlich auch in den Kartographischen Nachrichten. Es spricht auch nichts dagegen, ad hoc und zeitlich befristet Kommissionen zu aktuellen und speziellen Themen zu gründen, wenn eine kritische Masse an Akteurinnen und Akteuren zusammenkommt. Hierbei kann die DGfK ideell und finanziell genauso unterstützen wie beim immer wichtigeren Austausch mit anderen Disziplinen, z. B. den Gesundheits- oder Klimawissenschaften.

Vieles wird anders?! Vieles ist schon anders gewordenwir sollten uns die guten Dinge bewahren und nun die weniger guten verbessern. Was die DGfK betrifft: Mit „wir “ ist natürlich nicht nur der Vorstand gemeint, sondern alle Mitglieder und Kartographie-Begeisterten. Machen Sie also mit! Fangen Sie z. B. an, indem Sie mir Ihre Ansichten und Wünsche schreiben!

Zum Schluss dieser Nach-Corona-Betrachtungen aber auch noch dies: Ich freue mich auch wieder sehr auf ein gemeinsames Bier (je nach Region gerne auch auf ein Glas Wein) mit Ihnen!

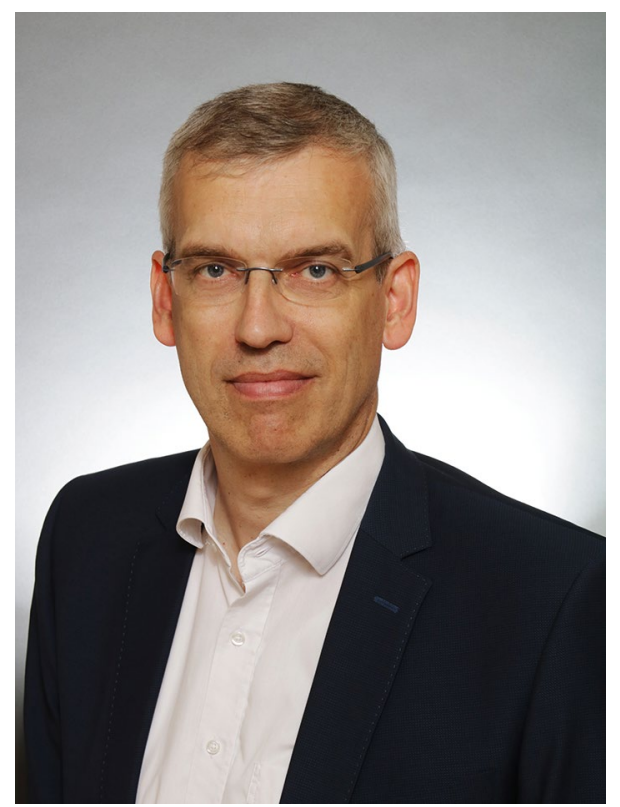

Supplementary Information The online version contains supplementary material available at https://doi.org/10.1007/s42489-021-00078-z.

Funding Open Access funding enabled and organized by Projekt DEAL.

Open Access This article is licensed under a Creative Commons Attribution 4.0 International License, which permits use, sharing, adaptation, distribution and reproduction in any medium or format, as long as you give appropriate credit to the original author(s) and the source, provide a link to the Creative Commons licence, and indicate if changes were made. The images or other third party material in this article are included in the article's Creative Commons licence, unless indicated otherwise in a credit line to the material. If material is not included in the article's Creative Commons licence and your intended use is not permitted by statutory regulation or exceeds the permitted use, you will need to obtain permission directly from the copyright holder. To view a copy of this licence, visit http://creativecommons.org/licenses/by/4.0/. 